\title{
The Future Radioactive Ion Beam Research Program at Oak Ridge
}

James B. Ball

Physics Division, Oak Ridge National Laboratory, Oak Ridge, Tennessee 37831, USA

\begin{abstract}
Early in 1995, the ORNL Holifield Facility is scheduled to return to operation as a dedicated radioactive ion beam (RIB) facility. This enhanced capability will open up new possibilities for research on the struciure of and phenomena occurring in proton-rich nuclei. Among the opportunities will be the creation of heavier $N \approx Z$ nuclei, approaching ${ }^{100} \mathrm{Sn}$, and the study of exotic nuclear shapes, extensions of studies of the $p-n$ interaction and superallowed beta decay, and examination of nuclear structure near the proton drip line. In addition to new nuclear and atomic physics research opportunities, the beams from the Holifield RIB facility are expected to provide new capabilities for measurements important to nuclear astrophysics. To carry out this experimental program, various upgrades are planned to the existing experimental apparatus, and a new, third-generation, recoil-mass separator is being constructed.
\end{abstract}

\section{INTRODUCTION}

For the past ten years, we have been operating the Holifield Facility at Oak Ridge as one of three heavy-ion accelerator facilities supported by the U.S. Department of Energy. As described by Olsen (Ref. 1), about a year ago we stopped operation of the experimental programs at Holifield to concentrate on reconfiguring our two accelerators to provide proton-rich radioactive ion beams. We expect to return to operation in 1995. At that time, we will be the only facility in North America dedicated to providing radioactive ion beams.

The research program supported by the Holifield RIB facility is expected to be concentrated almost exclusively on the topic of nuclear structure physics and nuclear astrophysics. In this paper, a little bit of the vision of what the early experimental program may look like, when full operation begins in a couple of years, is presented.

It is important to remember that an inherent limitation of this "first generation" RIB facility is that we are limited primarily to the production of proton-rich nuclei. It is this region, then, that will be the focus of the nuclear structure studies at Oak Ridge. For nuclear astrophysics, the principal concentration will likely be on those reactions and systems which govern the $r p$ process. The anticipated capabilities of the new facility are illustrated in Figure 1. 


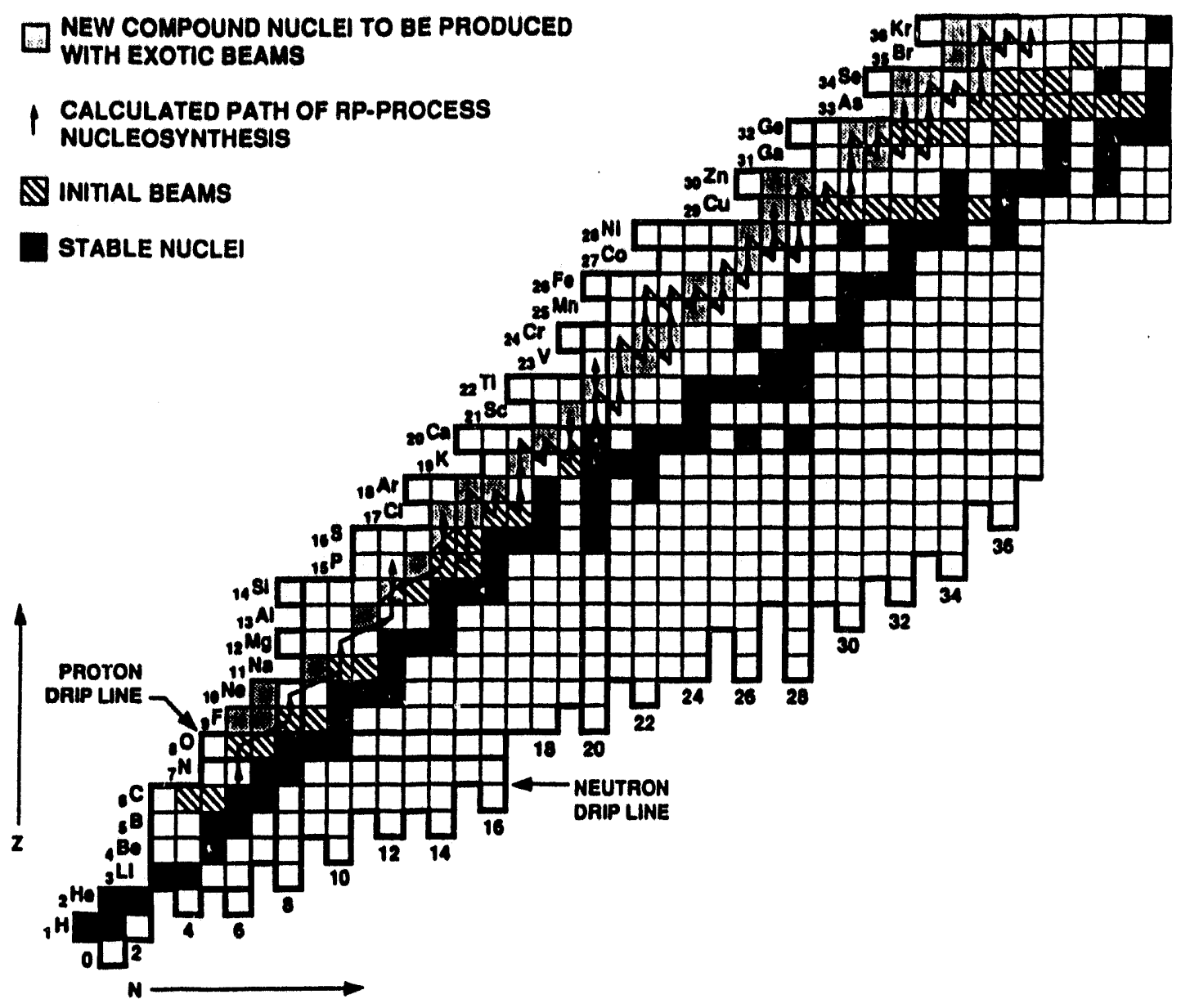

Figure 1. Planned initial beams and the "new" proton-rich compound nuclear systems that can be produced with the Holifield RIB Facility.

With these limitations in mind, the following are areas of research opportunities that are likely to be the areas of concentration for the early experimental program at the Holifield RIB Facility:

- structure of proton-rich nuclei

- studies of $N \approx Z$ nuclei

- tests of fundamental symmetries

- structure near the proton drip line

- nuclear astrophysics 
The remainder of this paper will deal briefly with each of these areas.

\section{STRUCTURE OF PROTON-RICH NUCLEI}

We expect that one of the major efforts will center on nuclear structure studies in those areas of proton-rich nuclei where there are theoretical predictions of large nuclear deformations. There are principally three such regions for study, where it is either very difficult or not possible to reach the nuclei of interest with stable beams on stable targets. These regions are in the very lightest masses for $Z=11$ to 19 , for $Z=33$ to 43 , and for $Z=56$ to 70. The first two of these regions are thought to be particularly gamma-soft and should have an interesting interplay between prolate and oblate deformations (Ref. 2). The possibility of large deformations in these nuclei, as well as new exotic shapes (oblate superdeformation, triaxial, nonaxial octupole) should highlight this program.

Of particular interest in the heavier regions of strong deformation will be the study of the neutron-proton interaction for high-j orbitals. In particular, the determination of the monopole residual $\mathrm{p}-\mathrm{n}$ interaction, and its role in driving deformations, can be much more clearly deduced in nuclei where the valance protons and neutrons are filling the same orbital.

\section{STUDIES OF $N \approx Z$ NUCLEI}

In addition to an in-depth study of the shape driving forces, as described above, the specific ability to extend the $\mathrm{T}=0$ nuclei higher in $\mathrm{Z}$ also holds much interest to the nuclear spectroscopist.

For many nuclear structure experimentalists, the long-sought-after 'El Dorado' has been

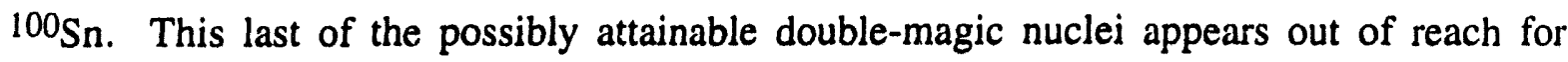
stable beams on stable targets.

This has been a region of nuclei in which the speaker has had a long-standing interest. Some 30 years ago we showed that the nuclei near $A=90$, where one is just beginning to populate the orbitals that make up this space, were particularly amenable to a simple shellmodel description. One indication of this was that by assuming a simple configuration for the low-lying levels in ${ }^{92} \mathrm{Nh}$, a trivial particle-hole transformation of this spectrum reproduced very well the low-lying levels in ${ }^{96} \mathrm{Nb}$. This same approach would suggest that the low-lying levels in ${ }^{100} \mathrm{In}$ should look like ${ }^{96} \mathrm{Nb}$ and that ${ }^{104} \mathrm{In}$ should closely resemble ${ }^{92} \mathrm{Nb}$. We know that the latter is not the case and we think we understand this in terms of the "pulling down" of the neutron $g_{7 / 2}$ orbital by the filling of the proton $g_{9 / 2}$ orbital. Hcwever, this may be a smaller effect at ${ }^{100}$ In and it will be very interesting to check this 
experimentally. In fact, from the shell-model standpoint, the observation of levels in the nuclei adjacent to ${ }^{100} \mathrm{Sn}$ that define the one- and two-body effective interactions are more interesting than $100 \mathrm{Sn}$ itself.

Will we be able to produce these nuclei? Figure 2 shows the region of the isotope chart around $A=100$. I've indicated on this plot those nuclei for which I have been able to find evidence for the observation of levels. Also shown are the compound systems that we will be able to create with radioactive beams that cannot be created with stable beams. From this plot it seems likely that we will be able to observe all the nuclei of interest, with the possible exception of ${ }^{100} \mathrm{Sn}$ itself. Although this may be possible, it also appears that this elusive nucleus will remain a significant challenge.

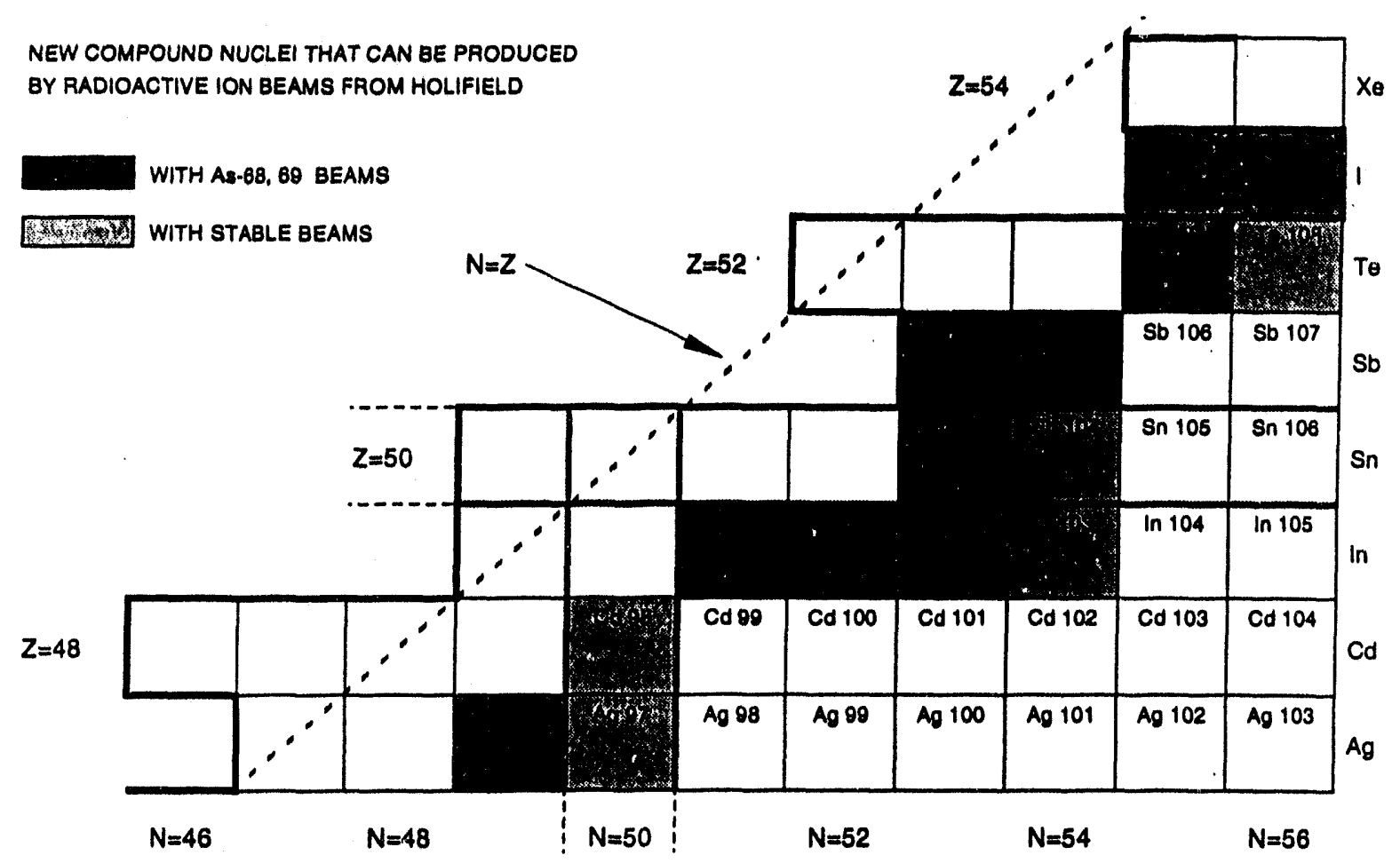

Figure 2. Nuclei presently observed in the $A=100$ region and the "new" compound nuclei that can be produced with radioactive beams.

\section{TESTS OF FUNDAMENTAL SYMMETRIES}

A comparison of the superallowed $\beta$ decay $(0+\rightarrow 0+$ decay between states of the same isospin) with $\mu$ decay currently provides the most sensitive test of the conserved vector current (CVC) hypothesis. Accurate measurements have been made for eight cases in a 
range of nuclear masses from $A=14$ to $A=54$. These data have been analyzed by Hardy et al. to provide a $0.04 \%$ test of $\mathrm{CVC}$, an uncertainty which is at the level of the quantum corrections to the standard model (Ref. 3).

The use of radioactive beams can extend the cases that can be studied, perhaps nearly to $A=100$. The importance of this is not just to provide additional cases, but to extend these measurements to nuclei with higher $Z$. Since the quantum corrections are $Z$ dependent, this may lead to a better understanding of these corrections. In addition, the higher $Z$ cases will provide, for the first time, the ability to measure the $0+$ to $0+$ isospin-forbidden and superallowed transitions in the same nucleus. This will provide a stringent test of the nuclear corrections, which involve the overlap of the mother and daughter wave functions. Thus, precise data from the heavier nuclear systems should increase the accuracy with which CVC can be tested.

\section{STRUCTURE NEAR THE PROTON DRIP LINE}

One of the first programs with the new radioactive beams at Holifield is expected to be the determination of the location of the proton drip line. At the present time, this has been delineated only to $\mathrm{Z} \approx 23$. The new beams will provide a substantial extension of this information.

These studies will supply mass information to compare with various model predictions. For example, the Möller-Nix formula includes a Coulomb redistribution term. This term is expected to be important for the very proton-rich nuclei.

In addition, these studies will provide spectroscopic data for nuclei beyond the proton drip line. At present, there are only eight cases which have been identified, but many more should be possible to produce with the new radioactive beams. The proton decay of these nuclei provide a new structure probe, and the competition between proton and $\alpha$-particle decays in the mass region around $\mathrm{Sn}$ and $\mathrm{Pb}$ may provide an important clue to understanding the preformation of $\alpha$ particles.

\section{NUCLEAR ASTROPHYSICS}

An indication of the nuclear astrophysics possibilities with the RIB facility can be seen in Figure 1, where the new beams and new compound nuclear systems to be produced are shown along with one calculation of the path of the $r p$ process (Ref. 4).

At the present time, studies of novae, supernovae, and X-ray bursts need $(p, \gamma),(\alpha, \gamma)$, and $(\alpha, p)$ capture reaction rates on proton-rich unstable nuclei. Some of these rates have been estimated from indirect studies, but most are unknown. Progress in quantitative 
understanding of these processes requires measurements with radioactive beams. Measurements of $(p, \gamma)$ and $(\alpha, \gamma)$ rates on unstable nuclei involved in the breakout from the hot $\mathrm{CNO}$ cycle, and in the $r p$ process, are expected to be among the first experiments with the new beams at Holifield.

\section{EXPERIMENTAL CONSIDERATIONS}

The experiments alluded to in the previous sections will require more than just providing radioactive beams. They will also place substantially increased demands on our experimental equipment.

The tandem accelerator is the best match to the DC properties of the ISOL-type source we will employ. While this has the distinct advantage of maximizing the beam intensity, those planning for experiments must remember that one has to give up the possibility of deriving timing from the beam. Thus, event timing for these experiments will need to be derived from other triggers. It must also be remembered that, even with this optimization, the "best" beams will still be low intensity by our present standards for stable beams.

With the beam intensities expected, conventional $\gamma$-ray techniques should be satisfactory for states populated with a cross section of 50 millibarns or larger, while a large detector array would be required to study superdeformed states. For the weaker channels (less than $100 \mu \mathrm{b}$ ), the use of a recoil-mass spectrometer (RMS) will be essential to providing mass identification. Other detector arrays, such as the charged-particle "dwarf ball," will be important in reducing spectra backgrounds.

One of the most important additions to the existing array of experimental devices at Holifield is the construction of a new, "third generation" RMS (Ref. 5). Shown in Figure 3, this device will be delivered in early 1994 and installed in a new experiment room.

Planning by the nuclear astrophysics community has also pointed to the need of a dedicated velocity-filter type device for this experimental program. Preliminary discussions have been held on the possibility of using the Daresbury RS for this device.

\section{SUMMARY}

We expect a very vigorous program of nuclear structure physics and nuclear astrophysics to capitalize on the availability of radioactive beams at the Holifield RIB Facility beginning in 1995. We look forward to resuming our operation for a worldwide user community. 


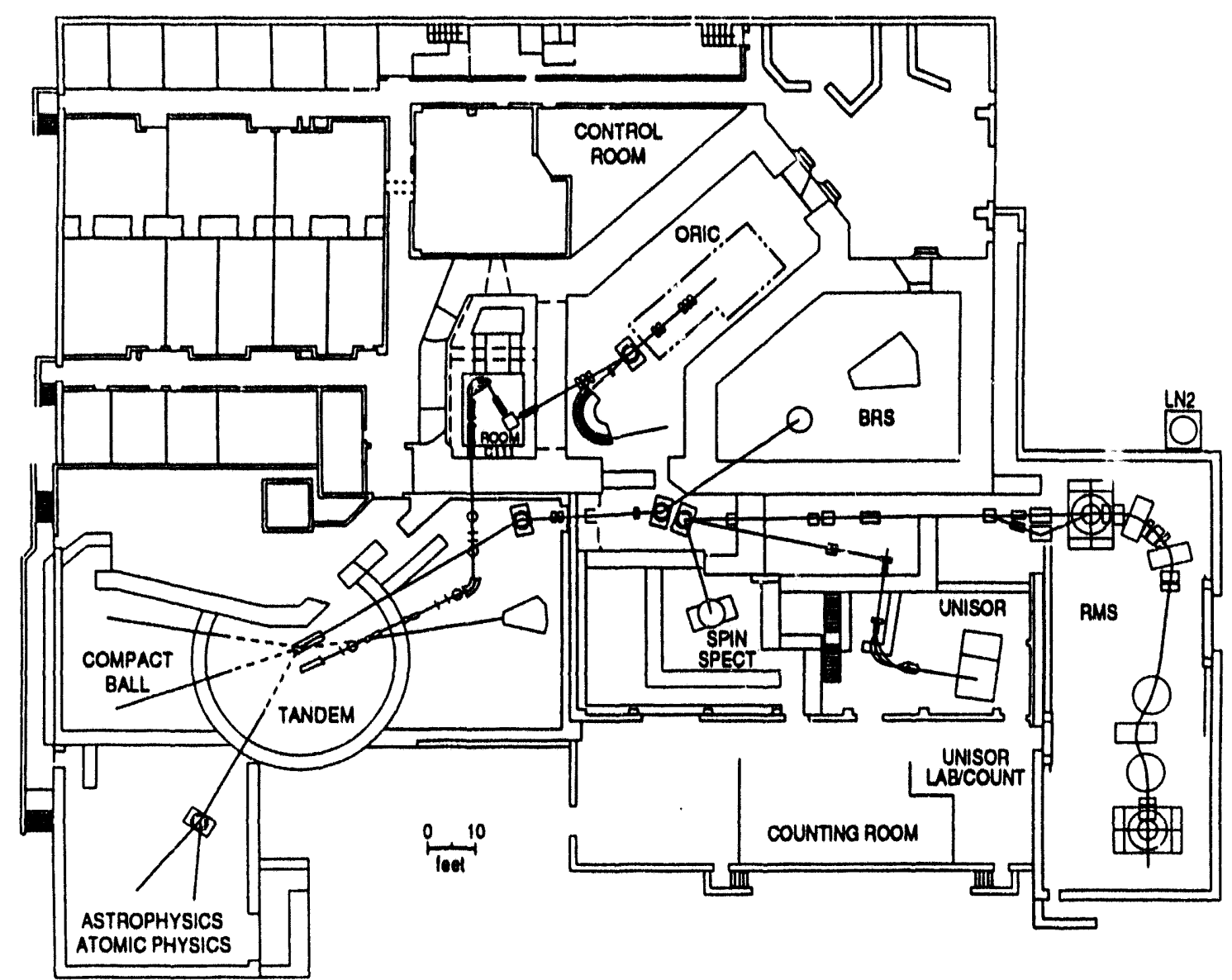

Figure 3. Layout of the Holifield RIB Facility accelerator and experiment areas. The new recoil-mass spectrometer and experiment area is shown at the far right.

More details about the facility and the planned research programs can be found in the original RIB proposal (Ref. 6) and in several subsequent papers and reports (Ref. 7,8). I would like to particularly thank Jerry Garrett, Ken Toth, Cyrus Baktash, and Michael Smith for helpful discussions during the preparation of this talk. Oak Ridge National laboratory is managed by Martin Marietta Energy Systems, Inc., for the U.S. Department of Energy under Contract No. DE-AC05-840R21400.

\section{REFERENCES}

1. D.K. Olsen, this conference

2. J. Dudek, in High-Spin Physics and Gamma-Soft Nuclei, ed. J.X. Saladin (World Scientific Press, Singapore, 1991) p. 146.

3. J.C. Hardy, I.S. Towner, V.T. Koslowsky, E. Hagberg, and H. Schmeing, Nucl. Phys. A509 (1990) 429.

4. M. Wiescher, J. Gorres, F.-K. Thielemann, and H. Ritter, Astron. Astrophys. 160 (1986) 56. 
5. T.M. Cormier, J.D. Cole, J.H. Hamilton, and A.V. Ramayya, Nucl. Inst. and Meth. A297 (1990) 199.

6. "A Proposal for Physics with Exotic Beams at the Holifield Heavy Ion Research Facility," ed. by J.D. Garrett and D.K. Olsen, Physics Division ORNL, February 1990.

7. J.D. Garrett, G.D. Alton, C. Baktash, D.K. Olsen, and K.S. Toth, Nucl. Phys. A557 (1993) $701 \mathrm{c}$.

8. D.K. Olsen, G.D. Alton, C. Baktash, H.K. Carter, D.T. Dowling, J.D. Garrett, D.L. Haynes, C.M. Jones, R.C. Juras, S.N. Lane, I.Y. Lee, M.J. Meigs, G.D. Mills, S. W. Mosko, B.A. Tatum, and K.S. Toth, in Radioactive Nuclear Beams, ed. Th. Delbar (Adam Hilger, 1992, London) p. 131. 
